\title{
Cochrane
}

Cochrane Database of Systematic Reviews

\section{Manual lymphatic drainage for lymphedema following breast cancer treatment (Review)}

Ezzo J, Manheimer E, McNeely ML, Howell DM, Weiss R, Johansson KI, Bao T, Bily L, Tuppo CM, Williams AF, Karadibak D

Ezzo J, Manheimer E, McNeely ML, Howell DM, Weiss R, Johansson KI, Bao T, Bily L, Tuppo CM, Williams AF, Karadibak D.

Manual lymphatic drainage for lymphedema following breast cancer treatment.

Cochrane Database of Systematic Reviews 2015, Issue 5. Art. No.: CD003475.

DOI: 10.1002/14651858.CD003475.pub2.

www.cochranelibrary.com 


\title{
[Intervention Review]
}

\section{Manual lymphatic drainage for lymphedema following breast cancer treatment}

Jeanette Ezzo ${ }^{1}$, Eric Manheimer ${ }^{2}$, Margaret L McNeely ${ }^{3}$, Doris M Howell ${ }^{4}$, Robert Weiss ${ }^{5}$, Karin I Johansson ${ }^{6}$, Ting Bao ${ }^{7}$, Linda Bily ${ }^{8}$, Catherine M Tuppo ${ }^{9}$, Anne F Williams ${ }^{10}$, Didem Karadibak ${ }^{11}$

${ }^{1}$ Research Director, JME Enterprises, Baltimore, Maryland, USA. ${ }^{2}$ Center for Integrative Medicine, University of Maryland School of Medicine, Baltimore, Maryland, USA. ${ }^{3}$ Department of Physical Therapy/ Department of Oncology, University of Alberta, Edmonton, Canada. ${ }^{4}$ Nursing, University Health Network (PMH site), Toronto, Canada. ${ }^{5}$ Porter Ranch, California, USA. ${ }^{6}$ Lymphoedema Unit, Department of Oncology, Skane University Hospital, Lund, Sweden. ${ }^{7}$ Integrative Medicine and Breast Cancer Services, Memorial Sloan Kettering Cancer Centre, New York, New York, USA. ${ }^{8}$ Stony Brook Medicine, New York, New York, USA. ${ }^{9}$ Bariatric and Metabolic Weight Loss Center, Stony Brook Medicine, Stony Brook, New York, USA. ${ }^{10}$ Queen Margaret University, Edinburgh, UK.

${ }^{11}$ School of Physical Therapy and Rehabilitation, Dokuz Eylul University, Izmir, Turkey

Contact address: Jeanette Ezzo, Research Director, JME Enterprises, 1905 West Rogers Ave, Baltimore, Maryland, 21209 , USA. jeanetteezzo@gmail.com.

Editorial group: Cochrane Breast Cancer Group.

Publication status and date: New, published in Issue 5, 2015.

Review content assessed as up-to-date: 24 May 2013.

Citation: Ezzo J, Manheimer E, McNeely ML, Howell DM, Weiss R, Johansson KI, Bao T, Bily L, Tuppo CM, Williams AF, Karadibak D. Manual lymphatic drainage for lymphedema following breast cancer treatment. Cochrane Database of Systematic Reviews 2015, Issue 5. Art. No.: CD003475. DOI: 10.1002/14651858.CD003475.pub2.

Copyright (C) 2015 The Cochrane Collaboration. Published by John Wiley \& Sons, Ltd.

\begin{abstract}
A B S T R A C T
Background

More than one in five patients who undergo treatment for breast cancer will develop breast cancer-related lymphedema (BCRL). BCRL can occur as a result of breast cancer surgery and/or radiation therapy. BCRL can negatively impact comfort, function, and quality of life (QoL). Manual lymphatic drainage (MLD), a type of hands-on therapy, is frequently used for BCRL and often as part of complex decongestive therapy (CDT). CDT is a fourfold conservative treatment which includes MLD, compression therapy (consisting of compression bandages, compression sleeves, or other types of compression garments), skin care, and lymph-reducing exercises (LREs). Phase 1 of CDT is to reduce swelling; Phase 2 is to maintain the reduced swelling.
\end{abstract}

Objectives

To assess the efficacy and safety of MLD in treating BCRL.

\section{Search methods}

We searched Medline, EMBASE, CENTRAL, WHO ICTRP (World Health Organization's International Clinical Trial Registry Platform), and Cochrane Breast Cancer Group's Specialised Register from root to 24 May 2013. No language restrictions were applied.

\section{Selection criteria}

We included randomized controlled trials (RCTs) or quasi-RCTs of women with BCRL. The intervention was MLD. The primary outcomes were (1) volumetric changes, (2) adverse events. Secondary outcomes were (1) function, (2) subjective sensations, (3) QoL, (4) cost of care. 


\section{Data collection and analysis}

We collected data on three volumetric outcomes. (1) LE (lymphedema) volume was defined as the amount of excess fluid left in the arm after treatment, calculated as volume in $\mathrm{mL}$ of affected arm post-treatment minus unaffected arm post-treatment. (2) Volume reduction was defined as the amount of fluid reduction in $\mathrm{mL}$ from before to after treatment calculated as the pretreatment LE volume of the affected arm minus the post-treatment LE volume of the affected arm. (3) Per cent reduction was defined as the proportion of fluid reduced relative to the baseline excess volume, calculated as volume reduction divided by baseline LE volume multiplied by 100 . We entered trial data into Review Manger 5.2 (RevMan), pooled data using a fixed-effect model, and analyzed continuous data as mean differences (MDs) with 95\% confidence intervals (CIs). We also explored subgroups to determine whether mild BCRL compared to moderate or severe BCRL, and BCRL less than a year compared to more than a year was associated with a better response to MLD.

\section{Main results}

Six trials were included. Based on similar designs, trials clustered in three categories.

(1) MLD + standard physiotherapy versus standard physiotherapy (one trial) showed significant improvements in both groups from baseline but no significant between-groups differences for per cent reduction.

(2) MLD + compression bandaging versus compression bandaging (two trials) showed significant per cent reductions of $30 \%$ to $38.6 \%$ for compression bandaging alone, and an additional 7.11\% reduction for MLD (MD 7.11\%, 95\% CI 1.75\% to 12.47\%; two RCTs; 83 participants). Volume reduction was borderline significant $(\mathrm{P}=0.06)$. LE volume was not significant. Subgroup analyses was significant showing that participants with mild-to-moderate BCRL were better responders to MLD than were moderate-to-severe participants.

(3) MLD + compression therapy versus nonMLD treatment + compression therapy (three trials) were too varied to pool. One of the trials compared compression sleeve plus MLD to compression sleeve plus pneumatic pump. Volume reduction was statistically significant favoring MLD (MD $47.00 \mathrm{~mL}, 95 \%$ CI $15.25 \mathrm{~mL}$ to $78.75 \mathrm{~mL}$; 1 RCT; 24 participants), per cent reduction was borderline significant $(\mathrm{P}=0.07)$, and LE volume was not significant. A second trial compared compression sleeve plus MLD to compression sleeve plus selfadministered simple lymphatic drainage (SLD), and was significant for MLD for LE volume (MD -230.00 mL, $95 \%$ CI - $450.84 \mathrm{~mL}$ to $-9.16 \mathrm{~mL}$; 1 RCT; 31 participants) but not for volume reduction or per cent reduction. A third trial of MLD + compression bandaging versus SLD + compression bandaging was not significant $(\mathrm{P}=0.10)$ for per cent reduction, the only outcome measured (MD $11.80 \%$, $95 \%$ CI $-2.47 \%$ to $26.07 \%, 28$ participants).

MLD was well tolerated and safe in all trials.

Two trials measured function as range of motion with conflicting results. One trial reported significant within-groups gains for both groups, but no between-groups differences. The other trial reported there were no significant within-groups gains and did not report between-groups results. One trial measured strength and reported no significant changes in either group.

Two trials measured QoL, but results were not usable because one trial did not report any results, and the other trial did not report between-groups results.

Four trials measured sensations such as pain and heaviness. Overall, the sensations were significantly reduced in both groups over baseline, but with no between-groups differences. No trials reported cost of care.

Trials were small ranging from 24 to 45 participants. Most trials appeared to randomize participants adequately. However, in four trials the person measuring the swelling knew what treatment the participants were receiving, and this could have biased results.

\section{Authors' conclusions}

MLD is safe and may offer additional benefit to compression bandaging for swelling reduction. Compared to individuals with moderateto-severe BCRL, those with mild-to-moderate BCRL may be the ones who benefit from adding MLD to an intensive course of treatment with compression bandaging. This finding, however, needs to be confirmed by randomized data.

In trials where MLD and sleeve were compared with a nonMLD treatment and sleeve, volumetric outcomes were inconsistent within the same trial. Research is needed to identify the most clinically meaningful volumetric measurement, to incorporate newer technologies in LE assessment, and to assess other clinically relevant outcomes such as fibrotic tissue formation.

Findings were contradictory for function (range of motion), and inconclusive for quality of life.

For symptoms such as pain and heaviness, $60 \%$ to $80 \%$ of participants reported feeling better regardless of which treatment they received. 
One-year follow-up suggests that once swelling had been reduced, participants were likely to keep their swelling down if they continued to use a custom-made sleeve.

\section{PLAIN LANGUAGE SUMMARY}

Manual lymphatic drainage for lymphedema following breast cancer treatment

\section{Background}

More than one in five of breast cancer patients will develop breast cancer-related lymphedema (BCRL). BCRL is a swelling that can occur in the arm, breast, or chest wall as a result of breast cancer surgery and/or radiation therapy. BCRL can negatively impact comfort, function, and quality of life

Manual lymphatic drainage (MLD) is a hands-on therapy that is commonly used for BCRL and often as part of complex decongestive therapy (CDT). CDT consists of MLD, compression bandaging, lymph-reducing exercises (LREs), and skin care.

\section{The Review Questions}

Is MLD safe and effective in treating BCRL?

\section{Study Characteristics}

We found six trials published through May, 2013, totaling 208 participants.

\section{Key Results}

When women were treated with a course of intensive compression bandaging, their swelling went down about 30\% to $37 \%$. When MLD was added to the intensive course of compression bandaging, their swelling went down another 7.11\%. Thus, MLD may offer benefit when added to compression bandaging.

Examining this finding more closely showed that this significant reduction benefit was observed in people with mild-to-moderate lymphedema when compared to participants with moderate-to-severe lymphedema. Thus, our findings suggest that individuals with mild-to-moderate BCRL are the ones who may benefit from adding MLD to an intensive course of treatment with compression bandaging. This finding, however, needs to be confirmed by further research.

When women were given a standard elastic compression sleeve plus MLD and compared to women who received a standard compression sleeve plus a nonMLD treatment, results were mixed (sometimes favoring MLD and sometimes favoring neither treatment.)

One-year follow-up suggests that once swelling had been reduced, participants were likely to keep their swelling down if they continued to use a custom-made sleeve.

MLD is safe and well tolerated.

Findings were contradictory for function (range of motion), with one trial showing benefit and the other not. Two trials measured quality of life, but neither trial presented results comparing the treatment group to the control, so findings are inconclusive.

No trial measured cost of care.

\section{Quality of the Evidence}

Trials were small ranging from 24 to 45 participants. Most trials appeared to randomize participants adequately. However, in four trials the person measuring the swelling knew what treatment the participants were receiving, and this could have biased results. 